\title{
Research on the Fault Diagnosis Method for Hoisting Machinery Based on Multi-source Information Fusion and BPNN
}

\author{
$\mathrm{Yi} \mathrm{Xie}^{1}$ and Jiangwen Zhang ${ }^{2}$ \\ ${ }^{1}$ School of Computer and Information Science, Southwest University, Beibei, Chongqing, 400715, China \\ ${ }^{2}$ Chong Qing Public Security Bureau Electronic Evidence Forensic Center, Yubei, Chongqing,401121, China
}

\begin{abstract}
In this paper, a fault diagnosis method for hoisting machinery based on multi-source information fusion and BPNN that has a fast training time and a high accuracy rate and can be converted to on-line monitoring system easily is provided. This method can be used to help people to real-time monitoring equipment and components and trace hazards. Compared with traditional methods currently used, the method has higher diagnostic accuracy and wider diagnostic range.
\end{abstract}

Keywords-multi-source information fusion; back propagation neural network; fault diagnosis; hoisting machinery introduction

With the advancement of China's modernization process, the hoisting machinery is widely used in infrastructure construction. The hoisting machinery plays an important role in today's society. Once the related accident occurs, it will cause great bodily injury and property damage. Therefore, it is necessary to research the fault diagnosis method for the hoisting machinery to reduce the occurrence of accidents [1].

However, the hoisting machinery actually is a kind of special equipment with most danger factors and biggest accident probability. The machine itself, the environment and the operators are potential hazards that may cause huge personal and property risk. Due to the special structure and movement forms, single-sensor cannot guarantee data acquisition normal. Only when multi-sensor information fusion is used, the reliable assessment of the equipment status can be ensured [2].

To solve the problem, a fault diagnosis method for the hoisting machinery based on multi-source information fusion is proposed to realize hazard identification and get the most objective and true security information. The specific algorithm uses the back propagation neural network as the basis algorithm and be realized using Matlab. The modeling method and algorithms are detailed in Section 2 and the implementation method is detailed in Section 3.

\section{METHODS}

\section{A. Multi-source Information Fusion}

Multi-source information fusion is the process of combining observations from a number of different sensors to provide a robust and complete description of an environment or process of interest [3]. Information fusion finds wide application in many areas of robotics, such as object recognition, environment mapping and localization [4]. Information fusion can provide numerous benefits in the several domains. The applications of data fusion are pervasive in robotics and underly the core problem of sensing, estimation and perception [5].Maintaining the Integrity of the Specifications

\section{B. Artificial Neural Network}

In machine learning and cognitive science, artificial neural networks (ANNs) are a family of models inspired by biological neural networks (the central nervous systems of animals, in particular the brain) and are used to estimate or approximate functions that can depend on a large number of inputs and are generally unknown [6]. Artificial neural networks are generally presented as systems of interconnected "neurons" which exchange messages between each other. The connections have numeric weights that can be tuned based on experience, making neural nets adaptive to inputs and capable of learning [7]

\section{Back Propagation Neural Network.}

Back Propagation Neural Network abbreviated as BPNN is a kind of neural network forms which has most applications currently. The BPNN is a systematic method of training multilayer artificial neural networks and is widely applied for pattern recognition, approximation and mapping of non-linear time-series prediction [8]. The BPNN includes [9,10]:

(1) An input layer with nodes representing input variables to the problem;

(2) An output layer with nodes representing the dependent variables;

(3) One or more hidden layers containing nodes to help capture the nonlinearity in the data.

The neurons between layers can be fully or partially interconnected between layers with weight (w). The structure of cases is shown in Figure 1.

\section{MODELING}

The hazards cause hoisting machinery accidents includes the apparatus body, personnel security, safety supervision, fault emergency and so on. In these hazards, the apparatus 
body hazard that is the main object of study in this paper needs multi-sensor to monitor the operational status. There are the main points that are monitored by multi-sensor as the following:

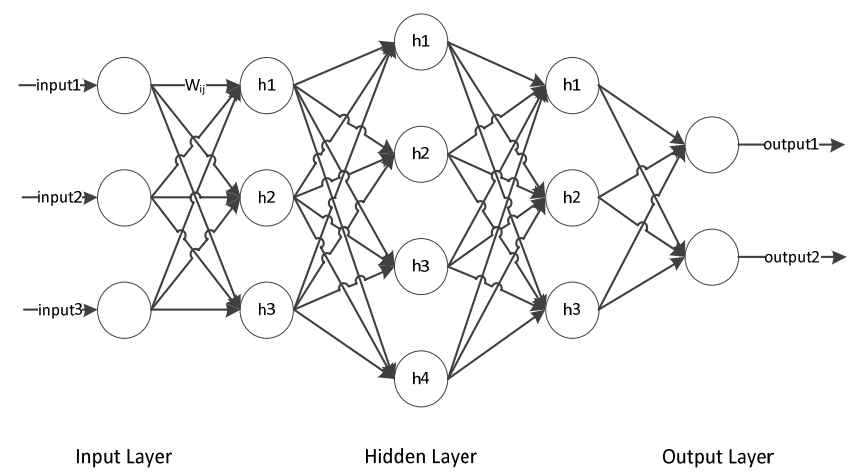

FIGURE I. THE STRUCTURE OF BACK PROPAGATION NEURAL NETWORK OF CASES

TABLE I. THE MONITORING TARGET

\begin{tabular}{|c|c|c|}
\hline$\#$ & The Monitoring Target & $\begin{array}{c}\text { The Number of } \\
\text { Sensors }\end{array}$ \\
\hline 1 & Overall stability & 24 \\
\hline 2 & Plastic Deformation & 18 \\
\hline 3 & Camber & 10 \\
\hline 4 & Deflection & 14 \\
\hline 5 & Hanging tube & 4 \\
\hline 6 & Steel wire & 6 \\
\hline 7 & Reel & 8 \\
\hline 8 & Pulley & 8 \\
\hline 9 & Brakes & \\
\hline
\end{tabular}

Due to the presence of multiple risk sources and multisensor, the fault diagnosis method likes Figure 2 uses two information fusion layers to make the evaluation results more objective and accurate. The back propagation neural network is used as the information fusion method and there are 10 BPNNs in this research.

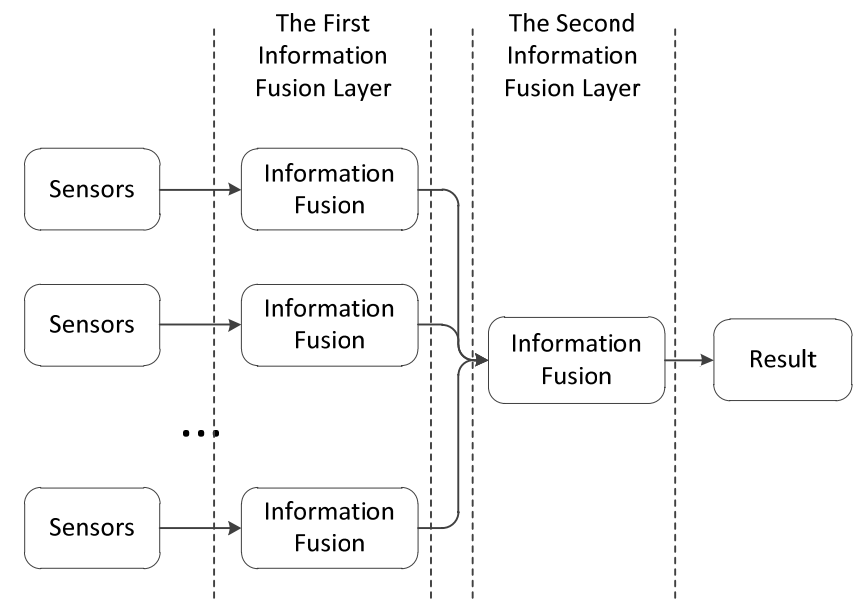

FIGURE II. THE STRUCTURE OF THE FAULT DIAGNOSIS METHOD

\section{ALGORITHM}

In the research structure, there are 10 BPNNs that the BPNNs in the first information fusion layer are defined as $n 1, n 2, \cdots, n 9$ and the BPNN in the second information fusion layer is defined as $n$.

The value of the $j_{\text {th sensor of the }} i$ th monitoring target is defined as $v_{i j}$. The $v_{i j}$ should be pre-processed by the MinMax Normalization method as following:

$$
v_{i j}=\frac{v_{i j}^{*}-\min _{\mathrm{i}}}{\max _{\mathrm{i}}-\min _{\mathrm{i}}}
$$

where $v_{i j}^{*}$ is the initial value of $v_{i j}$ without pre-process, $\min _{i}$ is the minimum value of the $i$ th monitoring target dataset, and $\max _{i}$ is the maximum value of the $i$ th monitoring target dataset.

In the first information fusion layer, the outputs of $n 1, n 2, \cdots, n 9$ are defined as following:

$$
O(n i)=f\left(v_{i 1}, v_{i 2}, \cdots, v_{i m}\right)
$$

where $i=1,2, \cdots, 9, m$ is the number of the sensors of $i$ th monitoring target, and $f(\bullet)$ is the processing of the BPNN algorithm.

And the final result $R$ is defined as following:

$$
R=O(n)=f(O(n 1), O(n 2), \cdots, O(n 9))
$$

And the result set contains four risk levels, which are:

- Stable

- Malfunction

- $\quad$ High Risk

- Mechanical Fatigue

Set the number of the hidden layer nodes of BPNN using the formula as following:

$$
x=\sqrt{x_{i}+x_{o}}+a
$$

where $x_{i}$ is the number of the input layer, $x_{o}$ is the number of the output layer, and $a$ is a constant between 1 and 10. The details of the 10 BPNNs is like as following: 
TABLE II.THE DETAILS OF THE 10 BPNNS

\begin{tabular}{|c|c|c|c|c|}
\hline$\#$ & $\begin{array}{c}\text { The 1st } \\
\text { Information } \\
\text { Fusion Layer }\end{array}$ & $\begin{array}{c}\text { The } \\
\text { Number of } \\
\text { Hidden } \\
\text { Layer } \\
\text { Nodes }\end{array}$ & $\begin{array}{c}\text { The 2nd } \\
\text { Information } \\
\text { Fusion Layer }\end{array}$ & $\begin{array}{c}\text { The } \\
\text { Number of } \\
\text { Hidden } \\
\text { Layer } \\
\text { Nodes }\end{array}$ \\
\hline 1 & $\mathrm{n} 1$ & 10 & & \\
\hline 2 & $\mathrm{n} 2$ & 9 & & \\
\hline 3 & $\mathrm{n} 3$ & 8 & & \\
\hline 4 & $\mathrm{n} 4$ & 9 & & \\
\hline 5 & $\mathrm{n} 5$ & 4 & & \\
\hline 6 & $\mathrm{n} 6$ & 6 & & \\
\hline 7 & $\mathrm{n} 7$ & 6 & & \\
\hline 8 & $\mathrm{n} 8$ & 8 & & \\
\hline 9 & $\mathrm{n} 9$ & 8 & & \\
\hline
\end{tabular}

IV. IMPLEMENTATION

The specific algorithm of the 10 BPNNs is detailed as following:

- Training algorithm: Levenberg-Marquardt (trainlm)

- Performance algorithm: Mean Squared Error (mse)

- Data division algorithm: Random (dividerand)

- Epoch maximum: 2000

- Performance goal: 0.01

- Validation Checks: 60

In $n 1$ case, the Performance graph and Training State graph are like as following and other BPNNs $n 2, n 3, \ldots ., n 9, n$ will not repeat.

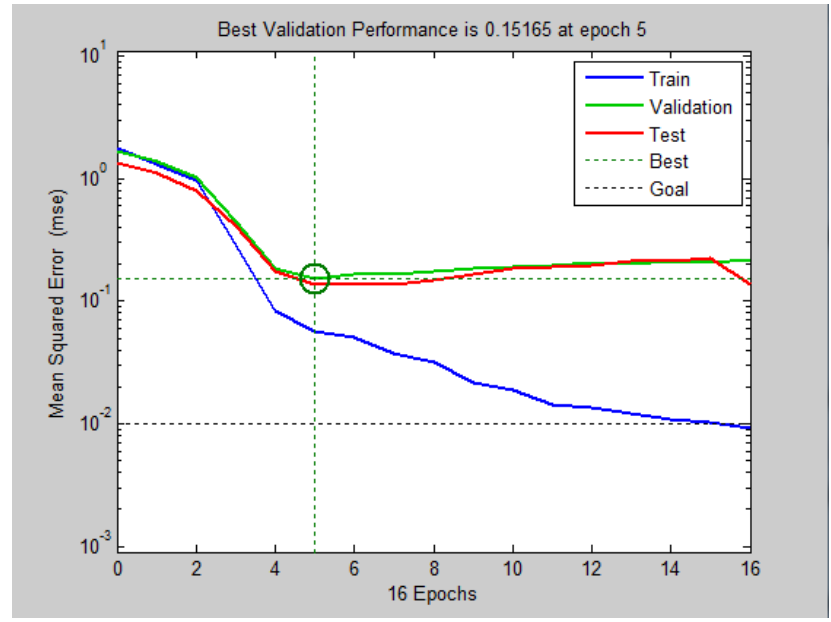

FIGURE III. PERFORMANCE

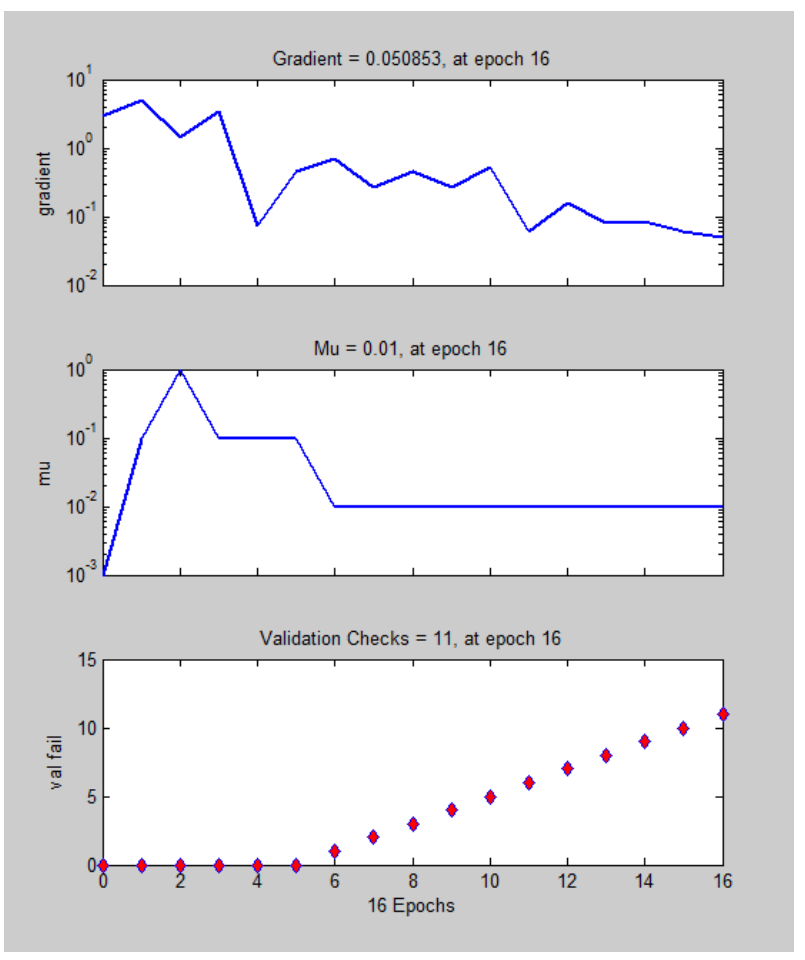

FIGURE IV. TRAINING STATE OF THE BPNN

Experiments show that the 10 BPNNs have a fast training time, and the fitting results can become the ultimate result through a simple rounding. After repeated training to 10 BPNNs, the overall fault diagnosis method has a $99.76 \%$ accuracy rate and has been proven to be effective in the detection of hoisting machinery failure. Compared with traditional methods, the fault diagnosis method can be converted to on-line monitoring system based on $\mathrm{B} / \mathrm{S}$ easily to achieve real-time fault diagnosis. Except detect the overall security situation, it can also detect components, so the fault diagnosis method can trace and track hazards.

\section{SUMMARY}

In this paper, a fault diagnosis method for hoisting machinery based on multi-source information fusion and BPNN that has a fast training time and a high accuracy rate and can be converted to on-line monitoring system easily is provided. This method can be used to help people to real-time monitoring equipment and components and trace hazards. Compared with traditional methods currently used, the method has higher diagnostic accuracy and wider diagnostic range.

\section{ACKNOWLEDGEMENTS}

Supported by "Fundamental Research Funds for the Central Universities", XDJK2015C112

\section{REFERENCES}

[1] LI Hui, XIA Qin, Safety Management Situation and Countermeasures of Construction Hoisting Machinery, The World of Building Materials, 34(2013)87-88. 
[2] GUO Hanzhu, WANG Mingzhong, Safety Actuality and Responsibility of Tower Crane Industry, Construction Machinery Technology \& Management, 8(2009)72-75.

[3] Baoping Cai, Yonghong Liu, Qian Fan, Yunwei Zhang, Zengkai Liu, Shilin Yu, Renjie Ji, Multi-source information fusion based fault diagnosis of ground-source heat pump using Bayesian network, Applied Energy, 114(2014)1-9.

[4] Jorge A. Balazs, Juan D. Velásquez, Opinion Mining and Information Fusion: A survey, Information Fusion, 27(2016) 95-110.

[5] João A. Duro, Julian A. Padget, Chris R. Bowen, H. Alicia Kim, Aydin Nassehi, Multi-sensor data fusion framework for $\mathrm{CNC}$ machining monitoring, Mechanical Systems and Signal Processing, 66-67(2016) 505-520.

[6] H.S. Wang, Y.N. Wang, Y.C. Wang, Cost estimation of plastic injection molding parts through integration of PSO and BP neural network, Expert Systems with Applications, 40(2) 418-428.

[7] Mingshun Li, Wencui Chen, Application of BP Neural Network Algorithm in Sustainable Development of Highway Construction Projects, Physics Procedia, 25(2012)1212-1217.

[8] Ying Wang, Cuijie Lu, Cuiping Zuo, Coal mine safety production forewarning based on improved BP neural network, International Journal of Mining Science and Technology, 25, 2(2015)319-324

[9] Xuefeng Zhao, Qin Ba, Lei Zhou, Weijie Li, Jinping Ou, BP neural network recognition algorithm for scour monitoring of subsea pipelines based on active thermometry, Optik - International Journal for Light and Electron Optics, 125, 18(2014) 5426-5431.

[10] Guolin Jing, Wenting Du, Yingying Guo, Studies on prediction of separation percent in electrodialysis process via BP neural networks and improved BP algorithms, Desalination, 291(2012) 78-93. 